\title{
A Six-Point Variant on the Lane-Riesenfeld Algorithm
}

\author{
Pakeeza Ashraf, ${ }^{1}$ Ghulam Mustafa, ${ }^{1}$ and Jiansong Deng ${ }^{2}$ \\ ${ }^{1}$ Department of Mathematics, The Islamia University of Bahawalpur, Bahawalpur 63100, Pakistan \\ ${ }^{2}$ Department of Mathematics, University of Science and Technology of China, Hefei 230026, China
}

Correspondence should be addressed to Ghulam Mustafa; ghulam.mustafa@iub.edu.pk

Received 30 November 2013; Accepted 15 April 2014; Published 8 May 2014

Academic Editor: Mohamad Alwash

Copyright (C) 2014 Pakeeza Ashraf et al. This is an open access article distributed under the Creative Commons Attribution License, which permits unrestricted use, distribution, and reproduction in any medium, provided the original work is properly cited.

We apply six-point variant on the Lane-Riesenfeld algorithm to obtain a new family of subdivision schemes. We also determine the support, smoothness, Hölder regularity, magnitude of the artifact, and the shrinkage effect due to the change of integer smoothing parameter that characterizes the members of the family. The degree of polynomial reproduction also has been discussed. It is observed that the proposed schemes have less shrinkage effect and as a result better preserve the shape of control polygon.

\section{Introduction}

The Lane-Riesenfeld algorithm [1] is a competent subdivision algorithm for subdividing uniform B-splines. To subdivide a B-spline curve, the algorithm is composed of two phases. The first phase doubles the control point by simply taking each control point twice. This step is followed by a sequence of smoothing operators which is midpoint averaging. By applying this subdivision process recursively, a sequence of piecewise linear curves is generated that in the limit converges to the uniform B-spline defined by the original control points. We may refer the articles [2-4] to the readers for further insight about subdivision schemes. However, for smoothness of the schemes, we may refer to [5-7].

In literature, there are different variants which are applied on the Lane-Riesenfeld algorithm. For example, Schaefer et al. [8] replaced the linear smoothing operator by nonlinear averaging rule in the Lane-Riesenfeld algorithm. They replaced the arithmetic mean with geometric mean to produce a subdivision scheme. Hormann and Sabin [9] offered a family of subdivision schemes by the convolution of uniform B-spline with kernel. Schaefer and Goldman [10] presented an algorithm for subdividing nonuniform B-splines of arbitrary degree in a manner similar to the Lane-Riesenfeld subdivision algorithm for uniform B-splines of arbitrary degree. Cashman et al. [11] presented generalized LaneRiesenfeld algorithm; they used same operator to define the refine and each smoothing stage. They applied four-point [12] variant on the Lane-Riesenfeld algorithm to introduce new family of schemes.

The best choice of smoothing operator is the one which keep the sense of balance among different properties of the schemes. For example, some of the smoothing operators give high degree of smoothness but very low degree of polynomial reproduction. So, choosing smoothing operator is a challenging task. One of the advantages of the proposed smoothing operator in this paper is that it keeps the balance among different properties of the schemes.

In this paper, we apply six-point variant on the LaneRiesenfeld algorithm to introduce family of subdivision schemes. Proposed family of schemes comprises two stages, that is, refine and smoothing, similar to the Lane-Riesenfeld algorithm, but the only difference is that we have used refine stage to introduce new points in the same way as each smoothing stage. We also show how the support, smoothness, Hölder regularity, magnitude of the artifact, and the shrinkage effect change with the change of integer smoothing parameter that categorizes the members of the family. Moreover, we 
find that each member of the family has quintic polynomial reproduction. We have the following findings.

(i) The proposed smoothing operator keeps a balance among different properties of the schemes. It also gives best degree of polynomial reproduction.

(ii) For the fixed number of control points, the number of smoothing stages is reciprocal to the magnitude of the artifacts.

(iii) Level of continuity of the schemes does not increase in general by increasing the smoothing stage. However, there are upper and lower bounds on Hölder continuity which grow gradually with the increase of smoothing stage.

(iv) The proposed schemes have less shrinkage effect and as a result better preserve the shape of control polygon.

(v) The smoothing stages affect the shrinkage in the limit curve while refine stages are not the factor of this effect.

\section{The $q$-Schemes}

Lane-Riesenfeld algorithm performs on an input sequence $P=\left\{p_{i}\right\}_{i \in Z}$ by using a refine stage $W$ followed by $m$ smoothing stages $S$. A subdivision step $Q$ is therefore $Q=$ $S^{m} W$, where $W=W_{L}$ is defined as refine stage in which each control point is doubled. $S=S_{L}$ is the smoothing stage in which midpoint averaging is used to compute new control points; that is,

$$
\left(S_{L} p\right)_{i}=\frac{1}{2}\left\{p_{i}+p_{i+1}\right\}
$$

The 6-point scheme [13] samples a local quintic interpolant to every 6 adjacent values just like the Lane-Riesenfeld smoothing operator $S_{L}$ which samples a local linear interpolant between every two adjacent values. This offers smoothing operator as

$$
\begin{aligned}
\left(S_{q} p\right)_{i}=\frac{1}{256}\{ & 3 p_{i-2}-25 p_{i-1}+150 p_{i}+150 p_{i+1} \\
& \left.-25 p_{i+2}+3 p_{i+3}\right\} .
\end{aligned}
$$

The new algorithm uses refine stage

$$
\begin{aligned}
\left(W_{q} p\right)_{2 i}= & p_{i} \\
\left(W_{q} p\right)_{2 i+1}=\frac{1}{256}\{ & 3 p_{i-2}-25 p_{i-1}+150 p_{i}+150 p_{i+1} \\
& \left.\quad-25 p_{i+2}+3 p_{i+3}\right\} .
\end{aligned}
$$

So, the subdivision operator for new family of schemes is given by

$$
Q_{q}=S_{q}^{m} W_{q},
$$

where refine stage $W_{q}$ is pursued by $m$ smoothing stages $S_{q}$. We call this family of schemes as the $q$-schemes. When we take 0th smoothing stage, then it reduces to the novel sixpoint scheme [13]. By increasing the number of smoothing stages, that is, $m$, we get new subdivision schemes. We can easily derive mask of the $q$-schemes for different values of $m$ as follows.

The factored form of the smoothing operator $S_{q}$ and refine stage $W_{q}$ can be written as

$$
\begin{aligned}
& W_{q}(z)=\left(\frac{1+z}{2}\right)^{6}\left(\frac{3 z^{-2}-18 z^{-1}+38-18 z+3 z^{2}}{4}\right), \\
& S_{q}(z)=\left(\frac{1+z}{2}\right)\left(\frac{3 z^{-2}-28 z^{-1}+178-28 z+3 z^{2}}{128}\right) .
\end{aligned}
$$

So, it is clear from (4) that the symbol of the $q$-schemes with $m$ smoothing stages can be written as

$$
a(z)=\left(\frac{1+z}{2}\right)^{m+6} e(z)
$$

with

$$
e(z)=c(z)^{m} d(z)
$$

where

$$
\begin{aligned}
& c(z)=\frac{1}{128}\left\{3 z^{-2}-28 z^{-1}+178-28 z+3 z^{2}\right\}, \\
& d(z)=\frac{1}{4}\left\{3 z^{-2}-18 z^{-1}+38-18 z+3 z^{2}\right\} .
\end{aligned}
$$

In Table 1, we present the mask $\alpha_{m}^{N}$ and complexity of the proposed schemes corresponding to $m=0,1,2$ and 3 .

Remark 1 (support of the $q$-schemes). The basis function is the map from positions in the domain to the limit curve of the refinement corresponding to a single old vertex having a unit value and all the others zero.

Since the smoothing operator $S_{q}$ makes use of six adjacent values to insert a new one, the number of nonzero entries in the mask of $S_{q}$ is 6 and the number of nonzero entries in the mask of refine stage $W_{q}$ is 11; therefore, by following [14], we conclude that support width of basis limit function for $S_{q}$ and $W_{q}$ is 5 and 10 , respectively.

Since the mask of $q$-schemes is obtained by applying refine stage $W_{q}$ on initial data followed by $m$ smoothing stages $S_{q}$, then the support width of basis limit function for the $q$ schemes is $5 m+10$. In Table 1 , we present supports of some of the $q$-schemes. By applying the algebraic condition (14) and Lemma 4.2 of [15] on the symbol of $q$-schemes (6), we can prove that the degree of polynomial reproduction of each $q$ scheme is quintic.

\section{Continuity Analysis of the $q$-Schemes}

In this section, we make use of Laurent polynomial (symbol) formalism [16] to calculate integer class continuity of the $q$ schemes. Lower bounds on Hölder continuity are computed by using the following theorem and upper bounds are 
TABLE 1: Complexity, support, and mask of the $q$-schemes corresponding to different values of $m$, and here $N$ shows complexity of the schemes (i.e., 6-, 8-, 11-, and 13-point schemes) and $S$ stands for support width.

\begin{tabular}{|c|c|c|c|}
\hline$m$ & $N$ & $S$ & Mask \\
\hline 0 & 6 & 10 & $\alpha_{6}^{0}=\frac{1}{256}[3,0,-25,0,150,256,150,0,-25,0,3]$ \\
\hline 1 & 8 & 15 & $\alpha_{8}^{1}=\frac{1}{256^{2}}[9,-75,375,1075,-3375,-6723,17175,57075,57075,17175,-6723,-3375,1075,375,-75,9]$ \\
\hline 2 & 11 & 20 & $\begin{array}{l}\alpha_{11}^{2}=\frac{1}{256^{3}}[27,-450,4350,-16050,7775,283608,-135000,1798600,399750,9920100 \\
16223412,9920100,399750,1798600,-135000,283608,7775,-16050,4350,-450,27]\end{array}$ \\
\hline 3 & 13 & 25 & $\begin{aligned} \alpha_{13}^{3}=\frac{1}{256^{4}} & {[81,-2025,28350,-220350,1008900,-1087220,-8846550,42100950,68212925,} \\
& -277340325,-404933940,1216712500,3659334000,3659334000,1216712500,-404933940, \\
& -277340325,68212925,42100950,-8846550,-1087220,1008900,-220350,28350,-2025,81]\end{aligned}$ \\
\hline
\end{tabular}

calculated by using Rioul's method [7]. Moreover, exact upper bounds on Hölder continuity can also be derived by using Floater and Muntingh algorithm [5].

Theorem 2. The lower bound on the Hölder continuity of the limit curves produced by the q-schemes with $m$ smoothing stages is

$$
(m+6)-\log _{2}\left(1+10\left(\frac{15}{8}\right)^{m}\right)
$$

Proof. Since by (6)

$$
a(z)=\left(\frac{1+z}{2}\right)^{m+6} e(z)
$$

then by Rioul [7] the Hölder continuity of the limit curves is bounded from below by

$$
(m+6)-\log _{2}\|e\| .
$$

Since $c(z)$ and $d(z)$ both are alternating symbols (i.e., its even coefficients are nonnegative and its odd coefficients are nonpositive), then $e(z)=c(z)^{m} d(z)$ is also alternating and

$$
\|e\|=\max \left(e^{e}, e^{o}\right),
$$

where $e^{e}$ and $e^{o}$ are sum of even and odd coefficients of $e(z)$, respectively. Since by [11]

$$
\left(\begin{array}{l}
e^{e} \\
e^{o}
\end{array}\right)=\left(\begin{array}{ll}
c^{e} & c^{o} \\
c^{o} & c^{e}
\end{array}\right)^{m}\left(\begin{array}{l}
d^{e} \\
d^{o}
\end{array}\right)
$$

then

$$
\left(\begin{array}{l}
e^{e} \\
e^{o}
\end{array}\right)=\left(\begin{array}{rc}
\frac{23}{16} & -\frac{7}{16} \\
-\frac{7}{16} & \frac{23}{16}
\end{array}\right)^{m}\left(\begin{array}{c}
11 \\
-9
\end{array}\right) .
$$

By eigenvalue decomposition, we have

$$
\left(\begin{array}{l}
e^{e} \\
e^{o}
\end{array}\right)=\frac{1}{2}\left(\begin{array}{cc}
-1 & 1 \\
1 & 1
\end{array}\right)\left(\begin{array}{cc}
\frac{15}{8} & 0 \\
0 & 1
\end{array}\right)^{m}\left(\begin{array}{cc}
-1 & 1 \\
1 & 1
\end{array}\right)\left(\begin{array}{c}
11 \\
-9
\end{array}\right) .
$$

TABLE 2: Comparison of continuity of the $q$-schemes.

\begin{tabular}{cccc}
\hline \multirow{2}{*}{ Continuity } & \multicolumn{2}{c}{ Hölder continuity } \\
& & Lower bound & Upper bound \\
\hline 0 & 2 & 2.540 & 2.830 \\
1 & 3 & 2.696 & 3.246 \\
2 & 3 & 2.823 & 3.649 \\
3 & 3 & 2.935 & 4.040 \\
4 & 3 & 3.038 & 4.421 \\
5 & 4 & 3.137 & 4.795 \\
\hline
\end{tabular}

This implies that

$$
\left(\begin{array}{c}
e^{e} \\
e^{o}
\end{array}\right)=\left(\begin{array}{c}
1+10\left(\frac{15}{8}\right)^{m} \\
1-10\left(\frac{15}{8}\right)^{m}
\end{array}\right) .
$$

Thus, we have

$$
\|e\|=1+10\left(\frac{15}{8}\right)^{m} \text {. }
$$

Consequently, the Hölder continuity of the limit curves produced by the $q$-schemes with $m$ smoothing stages is bounded from below by

$$
(m+6)-\log _{2}\left(1+10\left(\frac{15}{8}\right)^{m}\right) \text {. }
$$

Table 2 summarizes the continuity analysis of the $q$ schemes. We observe that the order of continuities achieved by $q$-schemes is reasonable, but it does not increase by increasing smoothing stages. However, there are upper and lower bounds on Hölder continuity which grow gradually with $m$.

Remark 3. Actually, the anonymous reviewer's suggested smoothing operator $S_{q}=((1+z) / 2)((1+z) / 2)^{2}\left(\left(z^{-1}+3+\right.\right.$ 
TABLE 3: Limit stencils of the $q$-schemes.

\begin{tabular}{|c|c|}
\hline$m$ & Limit stencils \\
\hline 0 & $l_{0}=[1]$ \\
\hline 1 & $\begin{aligned} l_{1}= & {[0.00000012323,-0.00000041069,-0.000039800,-0.00088283,0.016736,-0.10701,0.59120,0.59120,-0.10701,0.016736,} \\
& -0.00088283,-0.000039800,-0.00000041069,0.00000012323]\end{aligned}$ \\
\hline 2 & $\begin{aligned} l_{2}= & {[0.000000000000026,0.00000000044399,0.000000023348,-0.0000024738,0.00023116,0.00086974,-0.0097205} \\
& 0.027417,0.96241,0.027417,-0.0097205,0.00086974,0.00023116,-0.0000024738,0.000000023348,0.000000023348 \\
& 0.00000000044399,0.000000000000026]\end{aligned}$ \\
\hline 3 & $\begin{aligned} l_{3}= & {[-0.000000000003485,0.0000000011645,0.00000031630,0.000014667,0.000074973,-0.0020474,0.020295,-0.11208} \\
& 0.59374,0.59374,-0.11208,0.020295,-0.0020474,0.000074973,0.000014667,0.00000031630,0.0000000011645 \\
& -0.000000000003485]\end{aligned}$ \\
\hline
\end{tabular}

z)/5) significantly improve the smoothness of the schemes. That is, we get $C^{2}, C^{6}, C^{8}$, and $C^{8}$ smoothness for $m=0,1,2$, and 3 , respectively, but with only linear degree of polynomial reproduction. While by using our proposed smoothing operator, we get up to quintic degree of reproduction with $C^{2}, C^{3}, C^{3}$, and $C^{3}$ smoothness for $m=0,1,2$, and 3 , respectively.

\section{Analysis of the Limit Curve}

In this section, we present limit behavior, artifact analysis, and shrinkage effect of the $q$-schemes.

4.1. Limit Behavior of the q-Schemes. The limit behavior of the subdivision scheme can be analyzed by examining the eigenstructure of subdivision matrix $M$. For a nondefective matrix $M$, if there is $n$ linearly independent eigenvectors $v_{j}$ corresponding to the eigenvalues $\mu_{j}$, then it is possible to diagonalize $M$ by transforming it by the eigenvectors and their inverse; that is, $M=R A R^{-1}$. If the subdivision curve is $C^{0}$ (i.e., if all the rows of $M$ sum to one, then $M$ will have one eigenvector consisting of all ones and at least one eigenvalue must be one. If $1=\mu_{0}>\mu_{j}$, then subdivision scheme is $C^{0}$.), then all the vertices in the local neighborhood $P^{\infty}$ will shrink to the same point and thus we obtain limit stencil of the subdivision scheme mathematically

$$
P^{\infty}=\lim _{j \rightarrow \infty} P^{j}=M^{\infty} P^{0}=R A^{\infty} R^{-1} P^{0} .
$$

The subdivision matrices for the $q$-schemes corresponding to the masks $\alpha_{6}^{0}, \alpha_{8}^{1}, \alpha_{11}^{2}$, and $\alpha_{13}^{3}$ presented in Table 1 have order $11,15,21$, and 23, respectively. The limit stencils of these $q$ schemes for some smoothing stages are presented in Table 3. By applying limit stencil on consecutive control points in local neighborhood, we get limit position of the central one. Moreover, these stencils are useful to compute the magnitude of artifacts presented in the polygon.

4.2. Artifact Analysis of the q-Schemes. An artifact is defined to be any characteristic which is undesirable and cannot be separated by movement of control points which means that the curve holds spatial frequencies above the Shannon limit [17] relative to the density of the control polygon because features of the spatial frequency below this limit are removable by movements of the control points [18]. By definition, spatial frequency is the reciprocal of the number of control points per cycle and artifact magnitude is the function of the spatial frequency. We measure the amount of artifact presented in the polygon after the first subdivision level by using the following strategy. First, take the product of symmetric mask/symbol with symmetric limit stencil/symbol and then represent this product as polynomial in $\sigma=(1+z) / 2 z^{1 / 2}$. Then, the magnitude of artifact can be calculated by substituting $\sin (\pi \omega / 2)$ for $\sigma$ in that polynomial, where $\omega$ is the spatial frequency. The magnitude of the artifact presented in the limit curve is given by $G(\omega)=$ $(1 / 2) H(\sin (\pi \omega / 2))$ with the understanding that the data is sampled from a sinusoid with $k=1 / \omega$ samples per cycle, where $k$ is the number of control points.

Theorem 4. The amount of artifact presented in the limit curve produced by the 6-point binary scheme (i.e., $m=0$ ) is

$$
G(\omega)=\frac{1}{2}\left\{12 \eta^{10}-30 \eta^{8}+20 \eta^{6}\right\}
$$

where $\eta=\sin (\pi \omega / 2)$.

Proof. By substituting $m=0$ in (6) and (7), we get 6point binary scheme whose symbol in symmetric form can be written as

$$
\begin{gathered}
a(z)=\frac{1}{256}\left\{3 z^{-5}-25 z^{-3}+150 z^{-1}+256\right. \\
\left.+150 z-25 z^{3}+3 z^{5}\right\} .
\end{gathered}
$$

This can be manipulated as

$$
a(z)=\frac{1}{256}\left\{\frac{3(1+z)^{10}}{z^{5}}-\frac{30(1+z)^{8}}{z^{4}}+\frac{80(1+z)^{6}}{z^{3}}\right\} \text {. }
$$

Since the symbol of the limit stencil $l_{0}$ (as given in Table 3 ) of 6 -point binary scheme is $l_{0}(z)=1 z^{0}$, this implies that

$$
\begin{aligned}
a(z) & =a(z) l_{0}(z) \\
& =\frac{1}{256}\left\{\frac{3(1+z)^{10} 2^{10}}{2^{10} z^{5}}-\frac{30(1+z)^{8} 2^{8}}{2^{8} z^{4}}+\frac{80(1+z)^{6} 2^{6}}{2^{6} z^{3}}\right\} .
\end{aligned}
$$




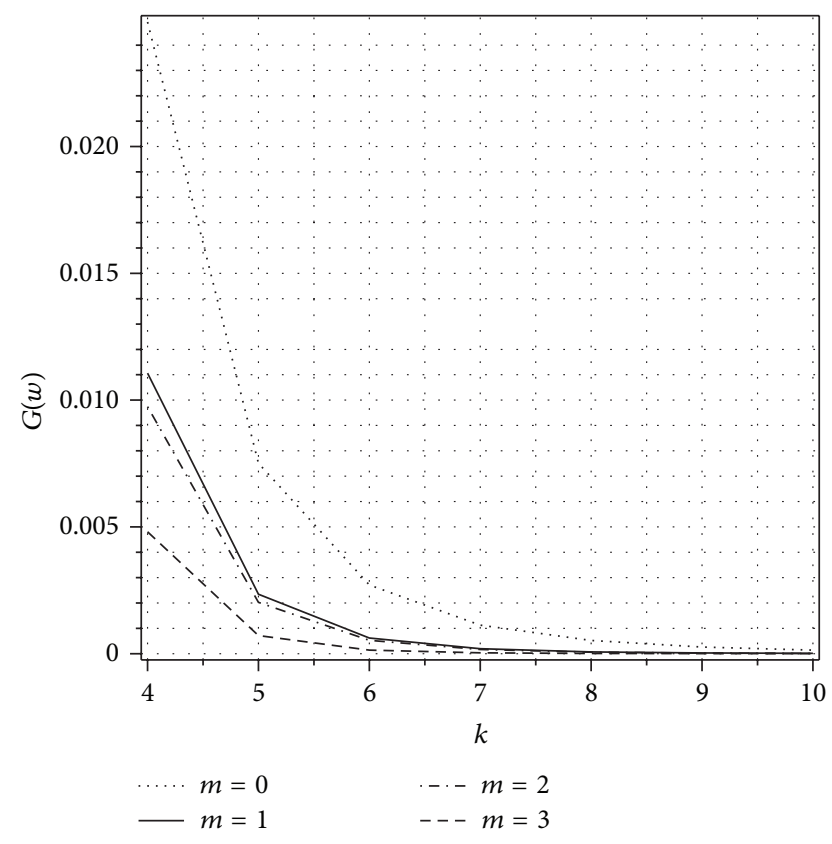

FIgURE 1: Artifact behavior of the $q$-schemes.

By representing it as polynomial in $\sigma=(1+z) / 2 z^{1 / 2}$, we get

$$
H(\sigma)=12 \sigma^{10}-30 \sigma^{8}+20 \sigma^{6} .
$$

Since the magnitude of the artifact presented in the limit curve is given by $G(\omega)=(1 / 2) H(\sin (\pi \omega / 2))$, then, by substituting $\sin (\pi \omega / 2)$ for $\sigma$ in above polynomial, we get

$$
G(\omega)=\frac{1}{2}\left\{12 \eta^{10}-30 \eta^{8}+20 \eta^{6}\right\}
$$

where $\eta=\sin (\pi \omega / 2), \omega=(1 / k)$, and $k$ is the number of control points of the polygon.

Similarly, we can prove the following theorems.

Theorem 5. The amount of artifact presented in the limit curve produced by the 8-point binary scheme (i.e., $m=1$ ) is

$$
\begin{aligned}
G(\omega)=\frac{1}{2}\{ & 0.00454 \eta^{28}-0.04505 \eta^{26}+0.11351 \eta^{24} \\
+ & 0.16850 \eta^{22}+0.9078 \eta^{20}-17.721 \eta^{18} \\
+ & 82.710 \eta^{16}-199.84 \eta^{14}+281.50 \eta^{12} \\
& \left.-220.79 \eta^{10}+75.003 \eta^{8}\right\} .
\end{aligned}
$$

Theorem 6. The amount of artifact presented in the limit curve produced by the 11-point binary scheme (i.e., $m=2$ ) is

$$
\begin{aligned}
G(\omega)=\frac{1}{2}\{ & 0.0000000028754 \eta^{36}+0.000012238 \eta^{34} \\
+ & 0.0000061307 \eta^{32}-0.0053021 \eta^{30} \\
+ & 0.15761 \eta^{28}-1.3285 \eta^{26}+5.6356 \eta^{24} \\
& -13.040 \eta^{22}+14.009 \eta^{20}+1.8945 \eta^{18} \\
& -8.3782 \eta^{16}-49.664 \eta^{14}+147.25 \eta^{12} \\
& \left.-156.89 \eta^{10}+62.358 \eta^{8}\right\} .
\end{aligned}
$$

Theorem 7. The amount of artifact presented in the limit curve produced by the 13-point binary scheme (i.e., $m=3$ ) is

$$
\begin{aligned}
G(\omega)=\frac{1}{2}\{ & -0.00000014453 \eta^{42}+0.000014495 \eta^{40} \\
& +0.00060407 \eta^{38}-0.0017688 \eta^{36} \\
& -0.042638 \eta^{34}+0.36221 \eta^{32}-0.76351 \eta^{30} \\
& -4.8648 \eta^{28}+45.306 \eta^{26}-191.67 \eta^{24} \\
& +534.46 \eta^{22}-1071.8 \eta^{20}+1583.8 \eta^{18} \\
& -1708.5 \eta^{16}+1288.1 \eta^{14}-611.30 \eta^{12} \\
& \left.+137.94 \eta^{10}\right\} .
\end{aligned}
$$

Similarly, we can determine the amount of artifact presented in the limit curves for other values of $m$. The amount of artifacts of the $q$-schemes for four (i.e., $m=0,1,2,3$ ) smoothing stages $S_{q}$ is shown in Figure 1. The magnitude of the artifact in the limit curve is plotted against the number of control points $k$. It is noticed that when the smoothing stages increase the magnitude of the artifact decreases for the same number of control points.

4.3. Shrinkage Effect of the q-Schemes. A control polygon is a sequence of points in space that is commonly used to manage the shape of an object. We apply subdivision scheme on closed control polygons to create visually smooth limit curves. Figure 2 shows limit curves generated by the $q$ schemes with different number of smoothing stages. Note that, as we increase the number of smoothing stages, final limit curve has larger distance from the control polygon. Since this shrinkage effect is only due to the smoothing stages, it is clear that the refine stage is not a factor of this effect. Therefore, we can measure this shrinkage effect by only examining smoothing stage. We can represent smoothing stage for a closed $k$-point polygon as the matrix

$$
\frac{1}{256}\left(\begin{array}{cccccccccc}
-25 & 150 & 150 & -25 & 3 & 0 & \cdots & 0 & 0 & 3 \\
3 & -25 & 150 & 150 & -25 & 3 & \cdots & 0 & 0 & 0 \\
0 & 3 & -25 & 150 & 150 & -25 & \cdots & 0 & 0 & 0 \\
\vdots & & \ddots & \ddots & \ddots & \ddots & \ddots & \ddots & & \vdots \\
-25 & 3 & 0 & 0 & 0 & 0 & \cdots & -25 & 150 & 150 \\
150 & -25 & 3 & 0 & 0 & 0 & \cdots & 3 & -25 & 150 \\
150 & 150 & -25 & 3 & 0 & 0 & \cdots & 0 & 3 & -25
\end{array}\right) \in \mathbb{R}^{k \times k}
$$




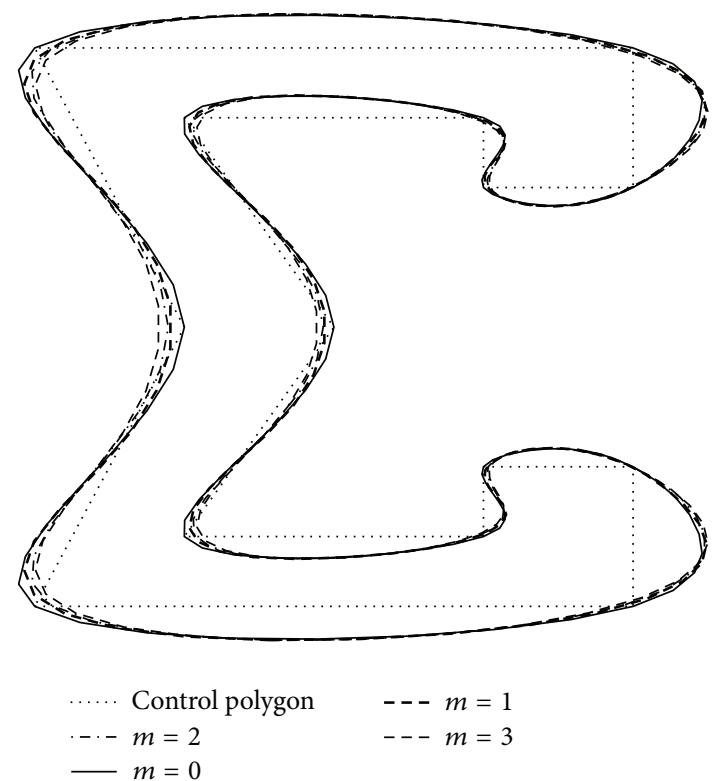

(a)

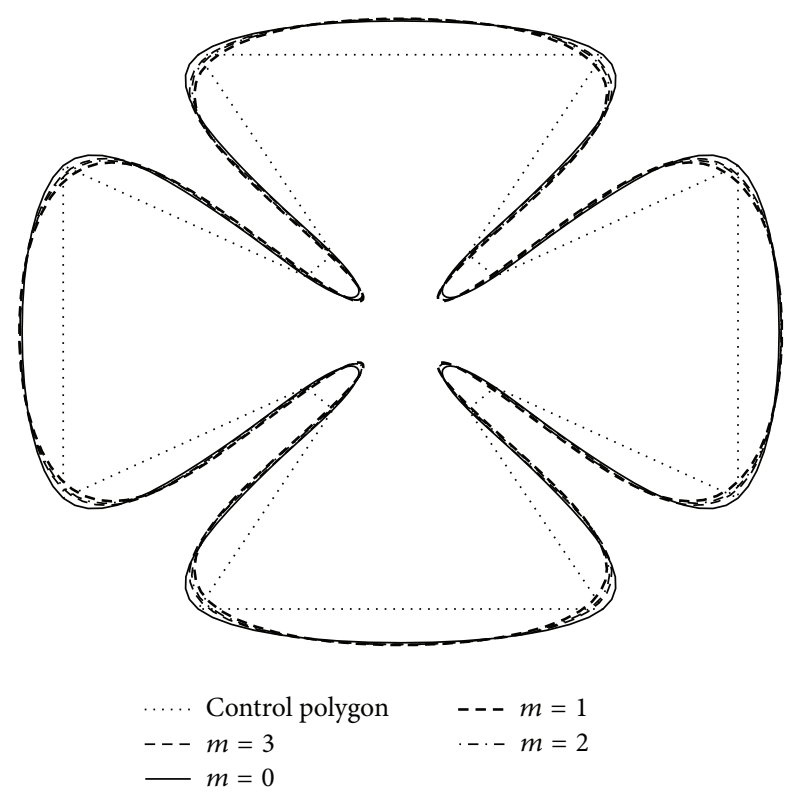

(b)

FIGURE 2: Limit curves generated by the $q$-scheme by applying $0,1,2$, and 3 smoothing stages.

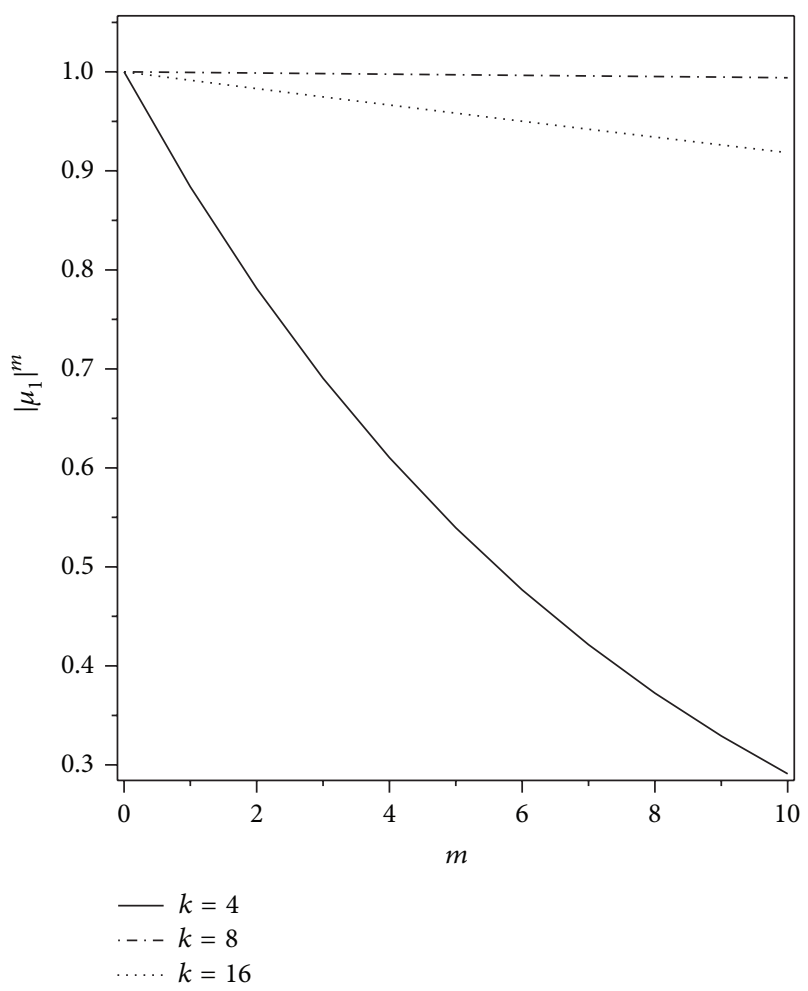

(a) C-schemes [11]

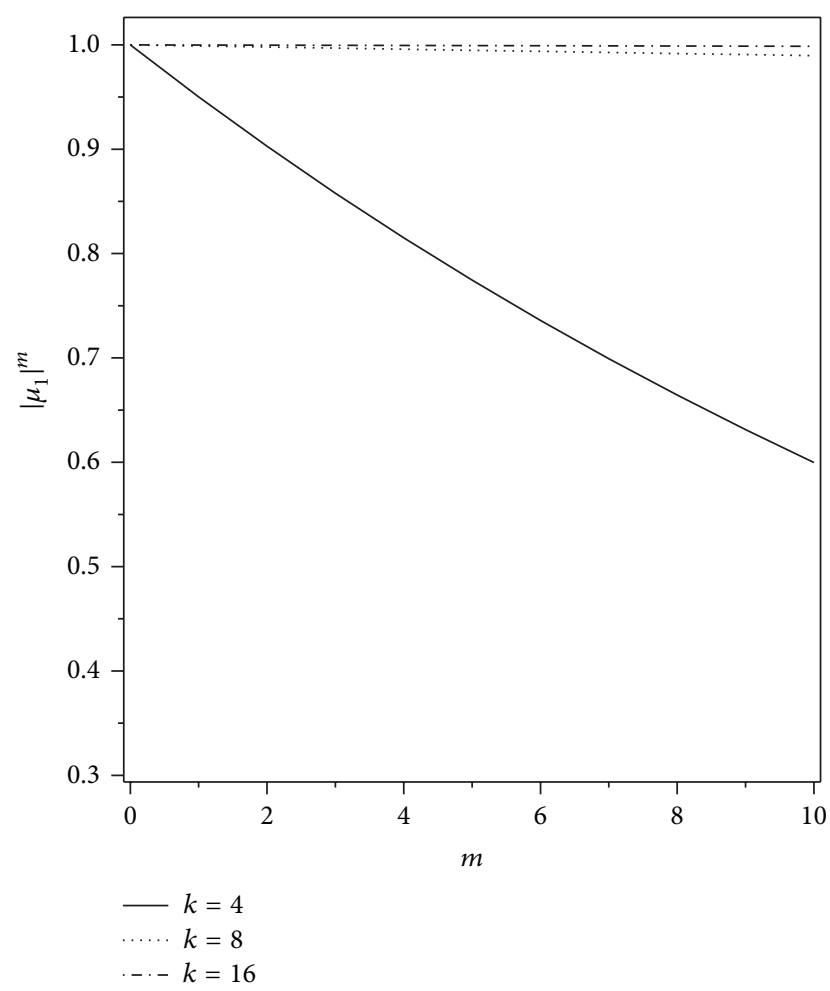

(b) proposed $q$-schemes

FIGURE 3: Rate of shrinkage plotted for the $C$-schemes [11] and proposed $q$-schemes against the number of smoothing stages $m$, for polygons having 4,8 , and 16 points. 
Since the above matrix is circulant, by [19], its eigenvalues are

$$
\begin{aligned}
\mu_{j}=\frac{1}{256}( & -25+150 e^{2 \pi i j / k}+150 e^{4 \pi i j / k}-25 e^{6 \pi i j / k} \\
& \left.+3 e^{8 \pi i j / k}+3 e^{-2 \pi i j / k}\right),
\end{aligned}
$$

where $j=0,1, \ldots, k-1$.

Here, we can determine that the dominant eigenvalue $\mu_{0}=1$ and its corresponding eigenvector is also a column vector consisting of all ones. This shows a dominant behavior that as we increase the smoothing stages, say $m \rightarrow \infty$, the whole configuration falls down towards barycenter of the original control polygon.

The subdominant eigenvalues $\mu_{1}$ and $\mu_{k-1}$ are complex conjugate of each other and shrinkage of the polygon towards its barycenter as well as a phase shift is decided by them. Disregarding this rotation, the rate of shrinkage for each smoothing stage is $\left|\mu_{1}\right|=\left|\mu_{k-1}\right|$. We plot this rate of shrinkage against the number of smoothing stages $m$ in Figure 3 . From Figure 3, it is apparent that the $q$-schemes bear less shrinkage when the number of smoothing stages increases and as a result better preserve the shape of control polygon as compared to $C$-schemes [11].

\section{Summary}

In this paper, we have applied 6-point variant on LaneRiesenfeld algorithm [1] to generate a new family of schemes, which we call the $q$-schemes. Furthermore, we have evaluated the relation among support, smoothness, Hölder continuity, and number of smoothing stages. The degree of polynomial reproduction also has been discussed. We have evaluated the rate of shrinkage of limit curve from original control polygon at different number of smoothing stages and make comparison with $C$-schemes [11]. Artifact and limit stencil analysis of proposed schemes is also carried out.

\section{Conflict of Interests}

The authors declare that there is no conflict of interests regarding the publication of this paper.

\section{Acknowledgments}

The authors thank the anonymous reviewers whose valuable comments greatly improved this paper. This work is supported by the Chinese Academy of Sciences (CAS) no. 2013FFJA0008, the NSF of China no. 11371341, and Indigenous $\mathrm{PhD}$ Scholarship Scheme of Higher Education Commission (HEC) Pakistan.

\section{References}

[1] J. M. Lane and R. F. Riesenfeld, "A theoretical development for the computer generation and display of piecewise polynomial surfaces," IEEE Transactions on Pattern Analysis and Machine Intelligence, vol. 2, no. 1, pp. 35-46, 1980.

[2] M. Aslam, G. Mustafa, and A. Ghaffar, "(2n-1)-point ternary approximating and interpolating subdivision schemes," Journal of Applied Mathematics, vol. 2011, Article ID 832630, 13 pages, 2011.

[3] G. Mustafa, J. Deng, P. Ashraf, and N. A. Rehman, “The mask of odd points $n$-ary interpolating subdivision scheme," Journal of Applied Mathematics, vol. 2012, Article ID 205863, 20 pages, 2012.

[4] G. Mustafa, P. Ashraf, and J. Deng, "Generalized and unifiedfamilies of interpolating subdivision schemes," Numerical Mathematics: Theory, Methods and Applications, vol. 7, 2014.

[5] M. S. Floater and G. Muntingh, "Exact regularity of symmetric univariate subdivision schemes," Geometry Seminar, Centre of Mathematics for Applications, University of Oslow, Oslo, Norway, 2012.

[6] R.-Q. Jia and Q. Jiang, "Spectral analysis of the transition operator and its applications to smoothness analysis of wavelets," SIAM Journal on Matrix Analysis and Applications, vol. 24, no. 4, pp. 1071-1109, 2003.

[7] O. Rioul, "Simple regularity criteria for subdivision schemes," SIAM Journal on Mathematical Analysis, vol. 23, no. 6, pp. 15441576, 1992.

[8] S. Schaefer, E. Vouga, and R. Goldman, "Nonlinear subdivision through nonlinear averaging," Computer Aided Geometric Design, vol. 25, no. 3, pp. 162-180, 2008.

[9] K. Hormann and M. A. Sabin, "A family of subdivision schemes with cubic precision," Computer Aided Geometric Design, vol. 25, no. 1, pp. 41-52, 2008.

[10] S. Schaefer and R. Goldman, "Non-uniform subdivision for Bsplines of arbitrary degree," Computer Aided Geometric Design, vol. 26, no. 1, pp. 75-81, 2009.

[11] T. J. Cashman, K. Hormann, and U. Reif, "Generalized LaneRiesenfeld algorithms," Computer Aided Geometric Design, vol. 30, no. 4, pp. 398-409, 2013.

[12] N. Dyn, D. Levin, and J. A. Gregory, "A 4-point interpolatory subdivision scheme for curve design," Computer Aided Geometric Design, vol. 4, no. 4, pp. 257-268, 1987.

[13] G. Deslauriers and S. Dubuc, "Symmetric iterative interpolation processes," Constructive Approximation, vol. 5, no. 1, pp. 49-68, 1989.

[14] I. P. Ivrissimtzis, M. A. Sabin, and N. A. Dodgson, "On the support of recursive subdivision," ACM Transactions on Graphics, vol. 23, no. 4, pp. 1043-1060, 2004.

[15] C. Conti and K. Hormann, "Polynomial reproduction for univariate subdivision schemes of any arity," Journal of Approximation Theory, vol. 163, no. 4, pp. 413-437, 2011.

[16] N. Dyn and D. Levin, "Subdivision schemes in geometric modelling," Acta Numerica, vol. 11, pp. 73-144, 2002.

[17] C. E. Shannon and W. Weaver, The Mathematical Theory of Communication, The University of Illinois Press, Champaign, Ill, USA, 1949.

[18] M. A. Sabin, U. Augsdörfer, and N. A. Dodgson, "Artifacts in box-spline surfaces," in Mathematics of Surfaces XI, vol. 3604 of Lecture Notes in Computer Science, pp. 350-363, Springer, Berlin, Germany, 2005.

[19] R. M. Gray, “Toeplitz and circulant matrices: a review, Foundations and Trends in Communications and Information Theory, vol. 2, no. 3, pp. 155-239, 2006. 


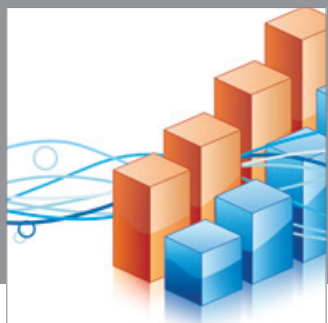

Advances in

Operations Research

mansans

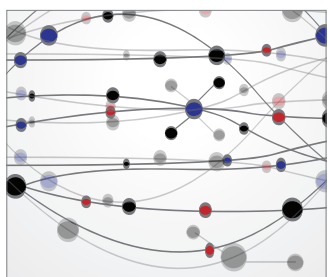

The Scientific World Journal
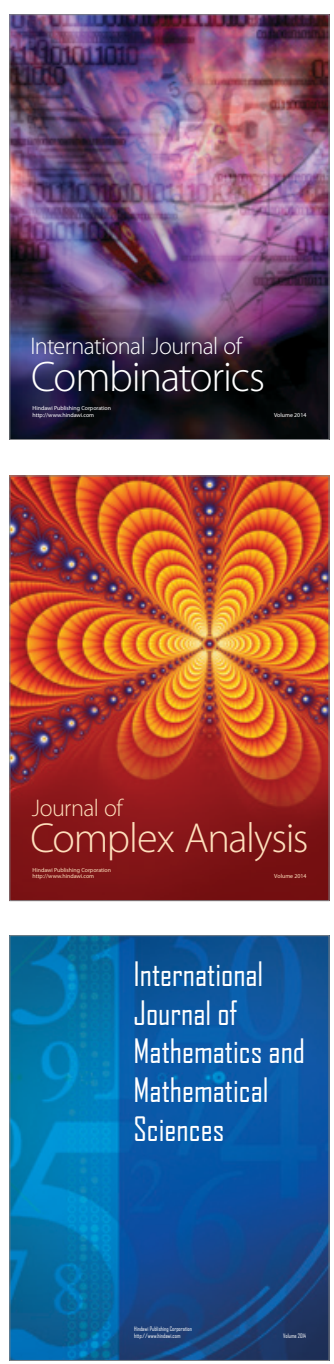
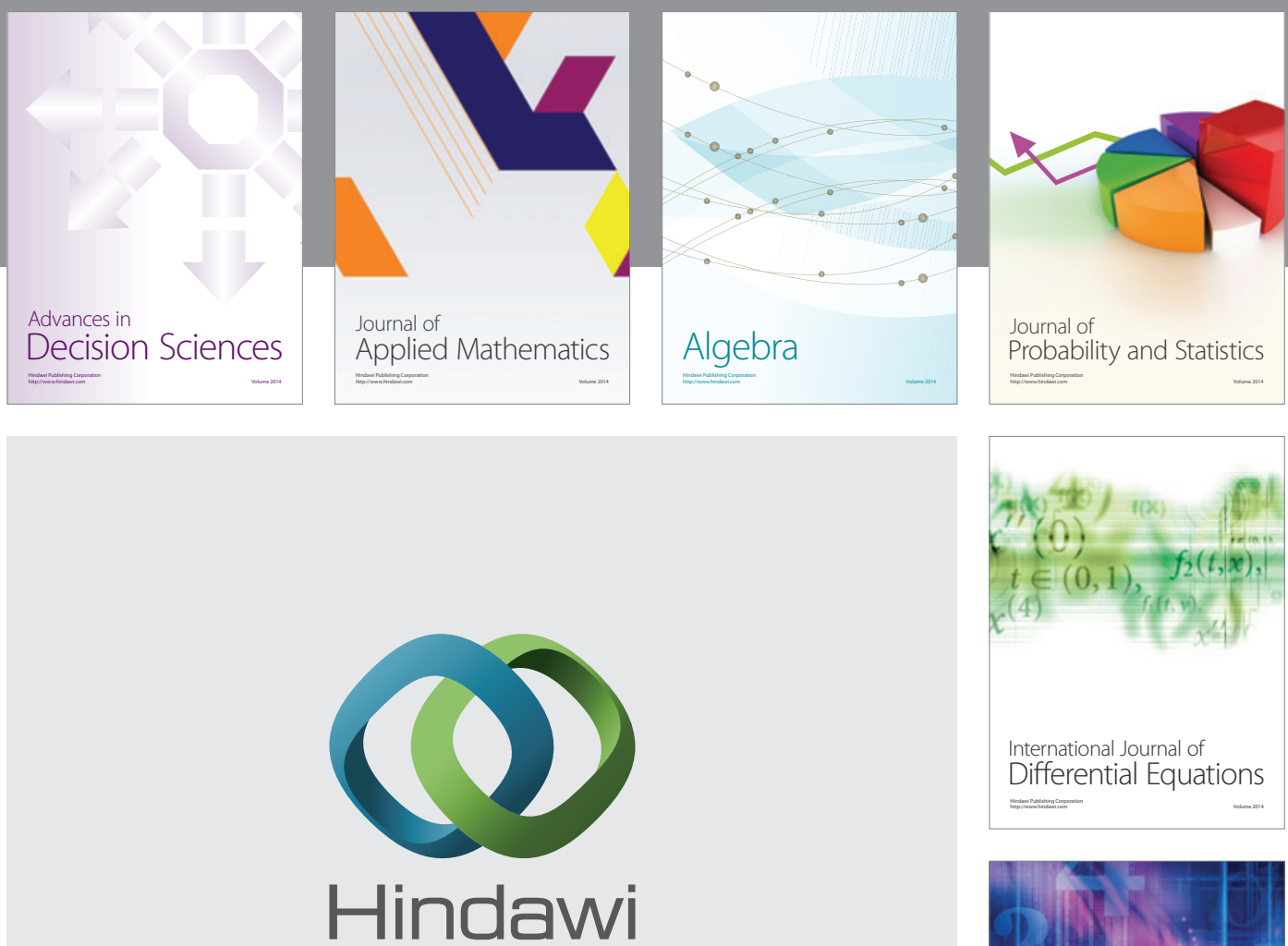

Submit your manuscripts at http://www.hindawi.com
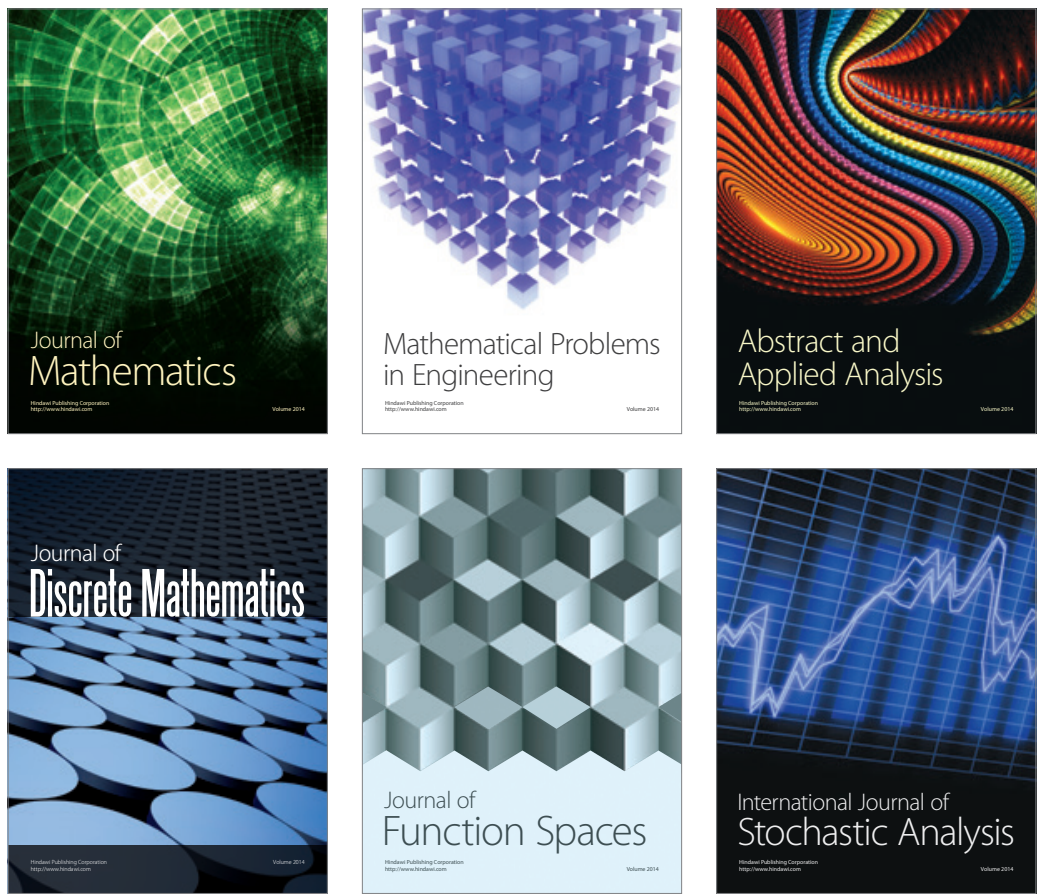

Journal of

Function Spaces

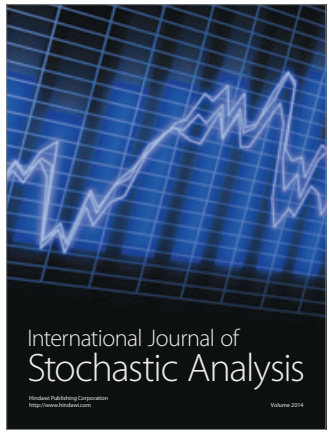

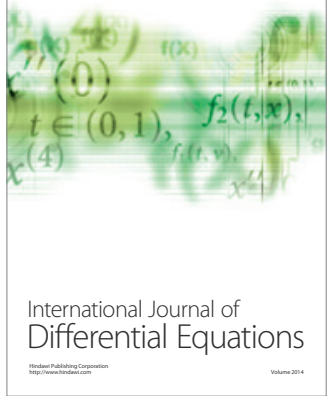
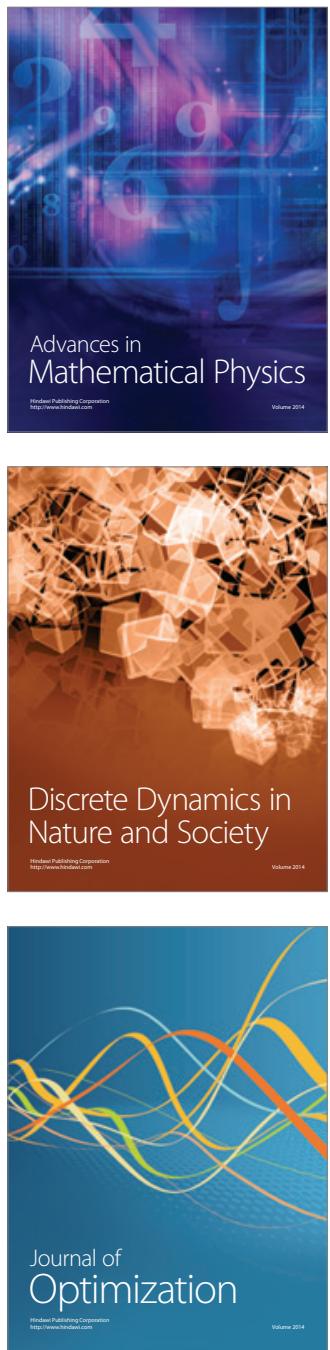